\title{
Review of exclusive enteral therapy in adult Crohn's disease
}

\author{
Nikola Mitrev @ $1,{ }^{1,2}$ Hin Huang, ${ }^{2}$ Barbara Hannah, ${ }^{1}$ Viraj Chandana Kariyawasam ${ }^{3}$
}

To cite: Mitrev N, Huang $\mathrm{H}$, Hannah B, et al. Review of exclusive enteral therapy in adult Crohn's disease. BMJ Open Gastro 2021;8:e000745. doi:10.1136/ bmjgast-2021-000745

- Additional supplemental material is published online only. To view, please visit the journal online (http://dx.doi. org/10.1136/bmjgast-2021 000745).

NM and HH contributed equally.

NM and $\mathrm{HH}$ are joint first authors.

Received 16 July 2021 Accepted 7 September 2021

Check for updates

(C) Author(s) (or their employer(s)) 2021. Re-use permitted under CC BY-NC. No commercial re-use. See rights and permissions. Published by BMJ.

${ }^{1}$ Department of Gastroenterology, Blacktown Hospital, Blacktown, New South Wales, Australia

${ }^{2}$ Western Sydney University Blacktown Mount Druitt Medical School, Blacktown, New South Wales, Australia

${ }^{3}$ Gastroenterology and Liver Services, Concord Hospital, Sydney, New South Wales, Australia

\section{Correspondence to} Dr Viraj Chandana Kariyawasam; viraj.kariyawasam@gmail.com

Dr Hin Huang; hinhuang3013@gmail.com

\section{ABSTRACT}

Background Exclusive enteral nutrition (EEN) is a potentially effective but underused therapy for Crohn's disease (CD) in adults. It is first-line induction treatment for paediatric patients but remains a second-line or thirdline therapy in adults.

Objective To analyse the evidence for EEN in adult patients with $\mathrm{CD}$, and summarise this in a narrative review. Methods In April/May 2020 and July 2021, a literature search was performed using the Medical Subject Headings (MeSH) terms: 'Crohn's disease', 'CD', 'inflammatory bowel disease', 'IBD', 'exclusive enteral nutrition', 'enteral nutrition', 'EEN', in PubMed, Scopus, Cochrane. Additional studies were obtained from references of search result articles as well as general reading. Studies with adult patients with CD treated with EEN were selected. 79 articles of relevance were found. Where data in adults were lacking, data from paediatric studies as extrapolated with care.

Results EEN in adult patients been shown to improve clinical, biomarker, endoscopic and radiologic measures of disease activity. EEN avoids the potential adverse effects of recurrent corticosteroids for induction such as metabolic derangements and opportunistic infections. EEN has also demonstrated benefits among adult patients with fistulising and stricturing CD. It may avoid surgery in such patients. Preoperative EEN has also been shown to reduce postoperative complications and recurrence. There appears to be benefits in combing EEN with antitumour necrosis factor agents, however, benefits of combination therapy with other biologics are less clear. A major drawback of EEN therapy in adults has been poor compliance. More palatable polymeric formulations improved patient education and dietitian support may overcome this. Evidence in adults is limited to small studies, often with suboptimal control arms and lack of blinding. Larger scale studies with improved study design are needed to confirm these beneficial effects.

Conclusion Despite limitations in evidence EEN should be considered in treating adults with CD.

\section{INTRODUCTION}

Crohn's disease (CD) is a chronic autoinflammatory disease of the gastrointestinal tract, caused by a complex interplay between genetic, gut microbiota and environmental factors. ${ }^{12} \mathrm{CD}$ has a worldwide prevalence of 3-20 per 100000 people with highest prevalence recorded in North America, Europe,
Australia and New Zealand. ${ }^{2}{ }^{3}$ Incidence is rising in Asia and South America. ${ }^{1}$ Peaks in occurrence are among teenagers, those in their 20-30s and those in their 50s. ${ }^{2}$

Current management of CD centres around inducing and maintaining remission. ${ }^{4}$ The European Crohn's and Colitis and the European Society for Paediatric Gastroenterology Hepatology and Nutrition guidelines recommend exclusive enteral therapy (exclusive enteral nutrition, EEN) as firstline induction treatment for $\mathrm{CD}$ affecting children. ${ }^{5}$ EEN provides all of the patient's nutritional requirements via premixed formulation given orally or via a nasogastric tube (NGT). ${ }^{6}$ EEN has similar or better clinical remission rates than corticosteroids in children, and also higher rates of endoscopic and histological remission. ${ }^{7-9}$ EEN also overcomes the negative effects of corticosteroids on bone mineral density, development and height attainment. ${ }^{1011}$

In contrast, adults with $\mathrm{CD}$ are usually given corticosteroids as a more convenient first-line induction treatment in a classic step-up approach. Many also practice a topdown approach, with early administration of biologics, such as infliximab or adalimumab, for induction of remission. The latest European Society for Clinical Nutrition and Metabolism guidelines recommend EEN in adults when steroids are not tolerated or contraindicated, in contrast to their first-line recommendation in children. ${ }^{12}$ Although effective in inducing clinical remission, longterm exposure to corticosteroids has potential adverse effects including diabetes, adrenal suppression, opportunistic infections and osteoporosis. ${ }^{13}$ EEN induction can overcome these issues however its uptake in management of adult $\mathrm{CD}$ varies around the world. Gastrointestinal Societies in UK and Australia have suggested that EEN may be considered in adult patients with $\mathrm{CD}$ where corticosteroid avoidance is sought. ${ }^{1415}$ This review will examine the evidence for EEN therapy in management of adult patients with CD. 


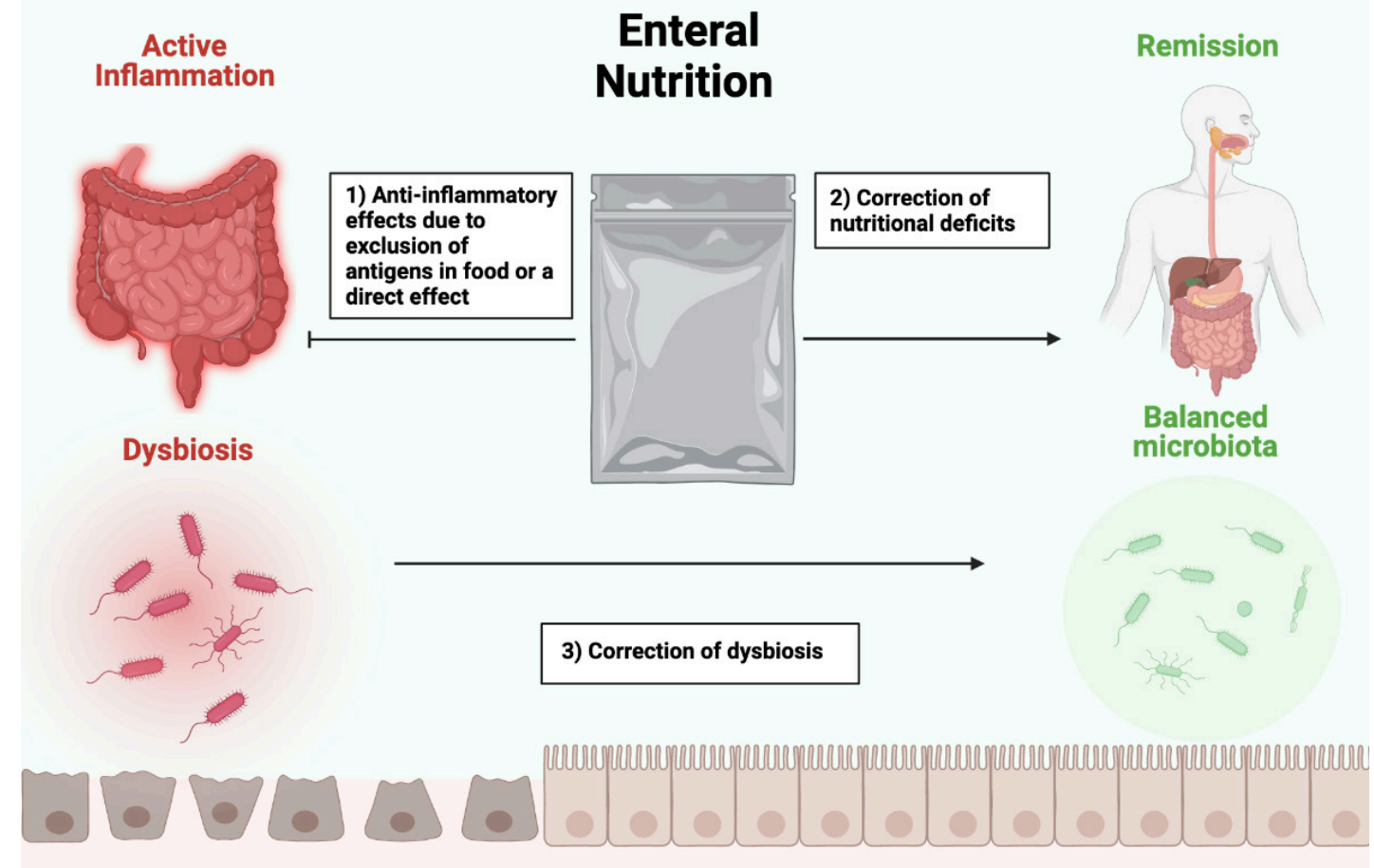

Figure 1 Mechanism showing the proposed effects of EEN. EEN improves outcomes in Crohn's disease patients by potentially: (1) reducing antigens in whole foods and/ or direct anti-inflammatory effects, ${ }^{34-38}$ (2) improving micronutrient and macronutrient deficiencies ${ }^{22-28}$ and (3) correcting gut microbiota disturbances. ${ }^{29-31} \mathrm{EEN}$, exclusive enteral nutrition.

\section{LITERATURE SEARCH}

PubMed, Cochrane and Scorpius databases were searched to avoid missing significant studies for this narrative review. The literature search was performed in May/April 2020 and July 2021 using the Medical Subject Heading (MeSH) terms 'Crohn's disease', 'CD', 'inflammatory bowel disease', 'IBD', 'exclusive enteral nutrition', 'EEN' and 'enteral nutrition'. Papers reporting on studies or systematic reviews, where EEN was part of an intervention, and the study population were adult patients with $\mathrm{CD}$, were selected. Studies published in languages other than English were excluded. Studies in paediatric populations were excluded, except when data in adults were insufficient. If evidence was extrapolated from paediatric to adult populations, limitations of this was acknowledged. Further articles were obtained from the references of searched articles and general reading. Overall, 79 studies were identified (online supplemental file 1).

\section{Proposed mechanism of action of EEN}

EEN improves outcomes in CD likely via nutritional repletion as well as direct and indirect anti-inflammatory effects (figure 1). There is potentially an interplay between nutritional status and active mucosal inflammation where improvements in one benefit the other. EENs influence on the gut microbiota may further reduce disease activity. ${ }^{16}$

\section{Improvement in nutritional deficiencies}

Nutritional deficiencies are found in $20 \%-85 \%$ of patients with CD. ${ }^{17}$ Mechanism may involve poor feeding habits, hypercatabolism from inflammation, malabsorption and side effects from treatment. Vitamin D deficiency can result in osteomalacia in children and osteopenia in adults. ${ }^{18}$ Stress fractures have been noted to occur in $22 \%$ of patients with $\mathrm{CD} .{ }^{19}$ Risk factors for malnutrition in $\mathrm{CD}$ includes fistulising disease and bowel surgery. ${ }^{18}$ Among inflammatory bowel disease (IBD) inpatients, malnutrition is associated with increased in-hospital mortality and length of stay. ${ }^{20}$

EEN improves nutritional parameters. A retrospective study examining elemental EEN prior to surgery found that 12/13 patients had weight gain and positive nitrogen balance. ${ }^{21}$ A retrospective observational study in adolescent patients demonstrated significantly greater weight gain with NGT administered EEN (median $9.2 \mathrm{~kg}$ ) compared with oral EEN (median $1.3 \mathrm{~kg}$ ).$^{22}$ Poor compliance with oral EEN may have contributed to these findings. A retrospective paediatric study found that EEN compared with corticosteroid significantly reduced linear growth failure and dependency on corticosteroids. ${ }^{23} \mathrm{EEN}$ may correct nutritional deficiencies through reducing inflammation-driven catabolism and promoting mucosal healing to improve nutrient absorption. Use of EEN also avoids corticosteroids which have negative effects on nutrition including fat gain, sarcopenia and osteomalacia. These nutritional studies have not been replicated in adult populations. Although EEN is also likely to improve weight and bone mineral density in adults, this needs to be further tested. 


\section{Microbiota changes}

Dysbiosis is implicated in the pathogenesis of IBD. ${ }^{24}$ Dysbiosis refers to an imbalance in the gut microbiota with reduced biodiversity and altered prevalence of certain bacterial species. Imbalances in Bacteroidetes, Firmicutes, Actinobacteria and Proteobacteria have been implicated in IBD pathogenesis and disease activity. In addition, several pathogenic species have been associated with development of $\mathrm{CD}$, including Campylobacter, Escherichia, Helicobacter and Mycobacterium. ${ }^{25}$

As diet is a major influence on gut microbiome composition, EEN is expected to alter it. A prospective cohort study compared the changes in gut microbiota after 8 weeks of EEN in six CD children, to temporal changes in the microbiome among healthy controls. ${ }^{26}$ After 8 weeks, there was a far greater change in the composition of the microbiota among patients with CD treated with EEN than was seen in healthy controls. Large changes in the Bacteroides composition and stability of Clostridium leptum correlated with decrease in the Paediatric Crohn's Disease Activity Index (CDAI). However, it is unclear which observed microbiota changes with EEN are the cause or the effect of reduced mucosal inflammation.

Two studies found that response to EEN is associated with changes in the microbiota that are distinct to microbiota changes observed in patients that enter remission without EEN. ${ }^{27} 28$ This suggests EEN may in part induce remission through modification of the gut microbiome. A reduction in certain bacterial species with EEN can remove some of the antigenic stimulus driving autoinflammation. This may explain the paradoxical observation of reduced microbial biodiversity associated with response to EEN. EEN response has also paradoxically been associated with relative reduction in bacteria that produce butyrate, a short chain fatty acid associated with gut health. ${ }^{29}$

\section{Anti-inflammatory effects}

EEN has several potential anti-inflammatory mechanisms in CD. Exclusion of dietary factors that trigger and potentiate inflammation may be important. ${ }^{30}$ Furthermore, EEN may increase expression of tight junction proteins between epithelial cells, reversing the increased gut permeability seen in CD. Exclusion of antigen triggers from reaching immune cells within the gut mucosa would reduce the inflammatory stimulus. ${ }^{31}$

EEN reduces systemic markers of inflammation. ${ }^{32-34} \mathrm{~A}$ prospective observational study on 32CD adults found after 2 weeks of EEN, median Harvey-Bradshaw Index (HBI) fell from 5 to 3 points $(p=0.003)$ and median serum $\mathrm{C}$ reactive protein (CRP) fell from 10 to $5 \mathrm{mg} / \mathrm{L}$ $(p=0.005) .{ }^{34}$ Similarly, a retrospective study of paediatric patients with $\mathrm{CD}$ found EEN resulted in reduction in mean CRP and increase in mean albumin and haemoglobin. ${ }^{32}$

\section{EN formulations}

EN formulations are divided into elemental or nonelemental. Elemental formulations contain free amino acids, and are generally less palatable. ${ }^{35}$ Non-element formulations are further subdivided in semielemental, containing short peptides from partially digested proteins and polymeric, containing undigested proteins. Nonelemental formulations are associated with better compliance and cost effectiveness. ${ }^{36} 37$ A Cochrane meta-analysis of 11 randomised controlled trials (RCTs) comparing elemental to non-elemental EEN found similar clinical remission rates between the two groups. ${ }^{38}$ Subgroup analysis between elemental, semielemental and polymeric feeds, showed similar efficacy. Similarly, there was no difference in clinical remission rates between low fat-content $(<20 \mathrm{~g} / 1000 \mathrm{kCal})$ and high fat-content EN formulas. However, very low-fat content and very low long chain triglycerides EN formulas were associated with higher clinical remission rates than those with higher content.

The composition of EN formulations varies considerably. A composition analysis of $61 \mathrm{EN}$ formulations with evidence for efficacy in CD found many contained additives that are implicated in CD onset, including modified starches, carrageenan, carboxymethyl cellulose and polysorbate $80 .{ }^{39}$ Clinical remission rates from RCTs in the latest Cochrane meta-analysis did not differ between studies utilising formulations with such additives compared with studies that did not contain such additives. Dietary interventions that have shown success may inform future EN formula development. However, the latest Cochrane review did not find compelling evidence for various diets for induction of remission in active CD. ${ }^{40}$

\section{EEN as induction therapy for adult patients with $C D$}

A Cochrane review found that potentially corticosteroids are more effective than EEN in adults for induction of clinical remission, but evidence was low quality (45\% vs $73 \%$, RR $0.65,95 \%$ CI 0.52 to 0.82$).{ }^{38}$ However, adverse effects of prolonged corticosteroids include osteoporosis, diabetes mellites, cataracts, depression and opportunistic infections. $^{13}$

Beyond clinical remission, EEN has superior rates of endoscopic healing than corticosteroids, a more objective treatment endpoint. Mucosal healing improves longterm outcomes in $\mathrm{CD}$ beyond clinical remission alone, by reducing risk of clinical flares and need for surgery. ${ }^{4142} \mathrm{~A}$ multicentre prospective study observed that endoscopic healing was achieved by $29 \%$ of patients with CD treated with prednisolone. ${ }^{43}$ In contrast, a prospective observational study found that 23/29 (73\%) of patients on EEN achieved mucosal healing. ${ }^{44}$ Mucosal healing rates between infliximab $(22 \%-60 \%)$ and short term EEN (33\%-58\%) were comparable. EEN appears highly effective in inducing mucosal healing in patients with CD. This study population was a mixture of adult and paediatric patients (average age 28.9 years). An RCT of paediatric patients found similar efficacy between EEN 


\begin{tabular}{|c|c|c|c|c|c|c|c|c|c|c|c|}
\hline \multirow[b]{2}{*}{ Ref } & \multirow[b]{2}{*}{ Year } & \multirow[b]{2}{*}{ Country } & \multicolumn{2}{|c|}{$\begin{array}{l}\text { No of } \\
\text { participants }\end{array}$} & \multirow{2}{*}{$\begin{array}{l}\text { Remission } \\
\text { criteria }\end{array}$} & \multicolumn{2}{|c|}{$\begin{array}{l}\% \text { achieving } \\
\text { remission (ITT) }\end{array}$} & \multirow[b]{2}{*}{$P$ value } & \multicolumn{2}{|c|}{$\begin{array}{l}\% \text { achieving } \\
\text { remission (PP) }\end{array}$} & \multirow{2}{*}{$\begin{array}{l}\text { \% EEN } \\
\text { discontinued - } \\
\text { unpalatable }\end{array}$} \\
\hline & & & EEN & CS & & EEN & CS & & EEN & CS & \\
\hline Engelman et $a /^{91}$ & 1993 & England & 7 & 4 & $\mathrm{HBI}<6.0$ & 100 & 100 & NS & 100 & 100 & 0 \\
\hline Gassull et al/92 & 2002 & Europe & 20 & 19 & VHAI $<120$ & 20 & 79 & 0.0005 & 27 & 79 & 25 \\
\hline Gassull et $a^{92}$ & 2002 & Europe & 23 & 19 & VHAI $<120$ & 52 & 79 & NS & 63 & 79 & 17 \\
\hline González-Huix et a $\left.\right|^{93}$ & 1993 & Spain & 15 & 17 & VHAI $<120$ & 8 & 88 & NS & 80 & 88 & 0 \\
\hline Gorard et $a l^{94}$ & 1993 & England & 22 & 20 & $\begin{array}{l}\text { HBI-remission } \\
\text { not defined, } \\
\text { mean<2 }\end{array}$ & 45 & 85 & $<0.05$ & 91 & 89 & 41 \\
\hline Lindor et $a l^{95}$ & 1992 & USA & 9 & 10 & $\begin{array}{l}\text { CDAl } \\
\text { decrease }>100 \\
\text { points }\end{array}$ & 50 & 33 & NS & 60 & 63 & 33 \\
\hline Lochs et $a l^{96}$ & 1991 & Europe & 55 & 52 & $\begin{array}{l}\text { CDAl } \\
\text { decrease }>100 \\
\text { points or }>40 \%\end{array}$ & 53 & 79 & $<0.01$ & 60 & 85 & 13 \\
\hline Malchow et $a l^{77}$ & 1990 & Europe & 51 & 44 & $\begin{array}{l}\text { CDAl } \\
\text { decrease }>100 \\
\text { points or }>40 \%\end{array}$ & 41 & 71 & $<0.05$ & 71 & 91 & 39 \\
\hline Mantzaris et $a l^{97}$ & 1996 & Greece & 10 & 10 & $\begin{array}{l}\text { CDAl }<150 \text { or } \\
\text { decrease }>100 \\
\text { points }\end{array}$ & 40 & 70 & NS & 40 & 70 & 0 \\
\hline Okada et $a^{98}$ & 1990 & Japan & 10 & 10 & $\mathrm{HBI}<1$ & 80 & 30 & $<0.01$ & 80 & 30 & 0 \\
\hline O'Morain et a ${ }^{99}$ & 1984 & England & 11 & 10 & $\begin{array}{l}\text { HBI-remission } \\
\text { not defined, } \\
\text { mean }<3\end{array}$ & 82 & 80 & NS & 100 & 100 & 18 \\
\hline Zoli et al ${ }^{100}$ & 1997 & Italy & 12 & 10 & $\mathrm{HBI}<3$ & 67 & 50 & NS & 80 & 60 & 8 \\
\hline
\end{tabular}

Adapted from wall et al ${ }^{6}$

CDAI, Crohn's Disease Activity Index; CS, corticosteroids; EEN, exclusive enteral nutrition; HBI, Harvey Bradshaw Index; ITT, intention to treat; NS, not significant; PP, per-protocol;

VHAI, Van Hees Activity Index.

and corticosteroids in inducing clinical remission. ${ }^{11}$ However, EEN had higher rates of mucosal healing (74\%) compared with corticosteroids (33\%, p<0.05). However, this may make results appear more favourable when extrapolated to adult patients.

Findings of studies comparing EEN to corticosteroids in adult patients with $\mathrm{CD}$ have varied. ${ }^{6}{ }^{34} \mathrm{~A}$ review of 11 studies comparing EEN to corticosteroids in adult patients with $\mathrm{CD}$, found clinical remission rates with EEN ranged from $8 \%$ to $100 \%$ (table 1 ).${ }^{6}$ Participant numbers in the EEN arm of each study were usually small, ranging from 7 to 55. The EEN and control group protocol for each study differed and no meta-analysis was performed. Discrepancies between the two treatment options could be attributed to compliance issues. ${ }^{6}$ Lower clinical remission rates with EEN in certain studies could be explained by high rates of non-compliance with EEN. Studies with high rates of compliance had similar rates of clinical remission with EEN and corticosteroids. Exclusion of studies with high non-compliance, however, may introduce selection bias similar to per-protocol analysis.

A meta-analysis by Schwab et al analysed results from 571 patients on elemental, oligopeptide and polymeric EEN. ${ }^{45}$ Clinical remission rates were $60 \%, 55 \%$ and $66 \%$, respectively, on an intention-to-treat basis, but on perprotocol analysis clinical remission rates were $73 \%, 70 \%$ and $67 \%$, respectively, similar to rates with corticosteroids. Studies in both paediatric and adult populations were pooled together in this meta-analysis, potentially making these results appear more favourable when extrapolated to adults. A recent observational study of 31 adult patients with CD treated with EEN demonstrated a statistically significant fall in the baseline CDAI at weeks 4 and 8 . However, almost two in three patients received steroids, immunomodulators and/or antitumour necrosis factor $\alpha$ (TNF $\alpha$ ) biologics in addition to the EEN. ${ }^{46}$

A common limitation of the above RCTs is in lack of blinding of patients. The fact that EEN replaces whole food diet makes blinding difficult. A placebo to EEN is not possible as it is the replacement of regular diet which is key. This differs to drug therapy trials, where placebo control and blinding can be achieved relatively easily. Unfortunately, there is no practical means of improving on current trial designs to achieve blinding.

EEN should be considered for induction when corticosteroids or other therapies are contraindicated or not favoured by the patients. An observational study of 12 female patients with CD who were pregnant or planning pregnancy, found that EEN was effective in inducing clinical remission in $83 \% .{ }^{47}$ Patients were intolerant of or had contraindications to corticosteroids, or preferred to trial EEN. This study was limited by small sample size and lack of control arm.

EEN retreatment may have reduced efficacy compared with first time use. ${ }^{14}$ A retrospective observational study in 52 paediatric patients with CD found higher clinical 
remission rates with first compared with second course of EEN (92\% vs $77 \%$ ). ${ }^{48}$ The difference between the two groups failed to reach statistical significance potentially due to the study being underpowered. Prior treatment failure also selects for a more aggressive disease phenotype. It is uncertain if this observation is true for adults and further studies are needed.

Fistulising and structuring complications of CD cause significant morbidity. EEN appears highly effective in fistulising and structuring $\mathrm{CD}$ in adults. In an observational study of 48 adult patients with CD with enterocutaneous fistulas treated with EEN, $63 \%$ achieved successful closure ${ }^{49}$ Lower CRP and higher body mass index (BMI) predicted for closure. Similarly, EEN achieved high rates of remission (94\%) and fistula closure (72\%) in another Japanese observational study. ${ }^{50}$ In a prospective observational study of 59 adult patients with $\mathrm{CD}$ with inflammatory small bowel strictures, on intention-to-treat analysis EEN achieved symptomatic remission in $81.4 \%$, radiologic remission in $53.8 \%$ and clinical remission in $64.6 \%{ }^{51}$ A prospective uncontrolled cohort study of 41 adult patients with $\mathrm{CD}$ with fistula, abscess or stricture formation treated with EEN found significant reduction in the CDAI ( $223.43 \pm 65.5$ vs $106.77 \pm 42.73$, $\mathrm{p} \leq 0.001)$, with $80.5 \%$ achieving full clinical remission. Closure of enterocutaneous fistulas was achieved in $75 \%$, intra-abdominal abscess resolved in $76 \%$ and $47 \%$ achieved mucosal healing. However, those with abscesses did receive antibiotics with or without percutaneous drainage. ${ }^{52}$

\section{EEN in perioperative patients with CD}

Some $30 \%$ of patients with CD would need surgery within 5 years of diagnosis and $80 \%$ would need surgery within 20 years of diagnosis. ${ }^{4}$ Corticosteroids have limited efficacy in stricturing and penetrative $\mathrm{CD}$ and surgery is often required. ${ }^{53}$ High-dose corticosteroid use presurgery is associated with perioperative infection and anastomotic breakdown. ${ }^{54}$

EEN has demonstrated efficacy in perioperative adult patients with CD. A meta-analysis of two prospective cohort studies found a significantly reduction in postoperative complications between those receiving preoperative-EEN $(21.9 \%)$, and those that did not $(73.2 \%$, $\mathrm{p}<0.001)$. The vast majority of the 831 patients in these pooled studies were adults. The number needed to treat was only 2, suggesting EEN was a highly effective intervention. ${ }^{5-57}$ However, both studies were conducted in Jinling hospital, China, with significant temporal overlap and likely many patients were represented in both studies. Although both studies found a benefit of preoperative EEN, performing a meta-analysis may not be appropriate. Most participants in these studies $(>90 \%)$ underwent open rather than laparoscopic surgery. This differs from Western practice; thus, caution should be exercised in extrapolation of benefit to Western countries. However, preoperative EEN has proven beneficial even with laparoscopic CD surgery. A cohort study of 120 adult patients with CD found that 4 weeks of EEN prelaparoscopic surgery improved albumin, haemoglobin and CRP presurgery, and also reduced postoperative complications $(\mathrm{p}<0.05) .{ }^{58}$

EEN for fistulising and structuring disease may avoid surgery. In a retrospective cohort study of 51 adult patients treated with EEN prior to surgery, 25\% avoided surgery altogether. ${ }^{59} \mathrm{~A}$ lack of prior EEN optimisation was associated with a ninefold increase in postoperative complications such as abscess formation and anastomotic leaks $(\mathrm{p}=0.04)$. Despite this promising result, the relatively small study cohort was from a single centre. A cohort study found that $\mathrm{EN}$ in adult patients with $\mathrm{CD}$ with an intra-abdominal abscess had a reduced need for surgery $(26.1 \%$ vs $56.3 \%, \mathrm{p}=0.01) .^{60}$ Multivariate analysis found $\mathrm{EN}$ to be an independent protective factor for requiring surgery. A recent Australian observational study found that among patients with fistulising and/ or structuring CD receiving EEN, a greater proportion in the group that completed at least 6 weeks of EEN avoided surgery $(100 \%)$ compared with those that did not complete the minimum of 6 weeks therapy $(43 \%$, $\mathrm{p}=0.02) .{ }^{61}$ Larger controlled trials with long-term data, however, are needed to confirm these findings.

Malnutrition presurgery increases risk of complications, and EEN can benefit this subset of patients. A small non-randomised prospective trial administering preoperative EEN to 10 malnourished adult patients with CD (defined as at least one of: BMI $<18.5 \mathrm{~kg} / \mathrm{m}^{2}$, serum albumin $<3 \mathrm{~g} / \mathrm{dL}$ or weight loss $>10 \%$ ). This significantly improved clinical disease activity, CRP and albumin, and avoided surgery in two patients. Despite the intervention population being malnourished, postoperative complications and 6-month recurrence rates were similar to five nutritionally replete patients who went to immediate surgery. ${ }^{16}$ Similarly, a prospective trial compared outcomes between high-risk patients who underwent EEN presurgery with a polymeric diet enriched with transforming growth factor-beta 2, to lowrisk patients who proceeded to direct surgery. ${ }^{62}$ Highrisk patients were those with any one of the following: presence of obstructive symptoms, steroid treatment, preoperative weight loss $>10 \%$ and perforating CD. Postoperative complication rates were similar between the high-risk and low-risk patients $(8 / 35$ (23.8\%) vs $5 / 21(22.9 \%), p=1)$. EEN presurgery may be of greatest benefit among malnourished or high-risk patients, improving outcomes to a similar level as nourished or lower-risk patients.

Finally, EEN may reduce postoperative recurrence. A retrospective study of adult patients with $\mathrm{CD}$ found that EEN for 4 weeks presurgery reduced infectious and noninfectious complications, as well as recurrence rates at 6 months but not at 2 years. ${ }^{63}$ Cohort studies have also found that postoperative EN may further reduce clinical and endoscopic recurrence postsurgery among adult patients with $\mathrm{CD} .{ }^{64-66}$ 


\section{EN as maintenance therapy}

On successful induction with EEN, several studies have demonstrated benefits of longer-term maintenance with cyclical or intermittent EN. Nocturnal EN in paediatric patients administered via NGT prolongs the remission period. A recent French study in paediatric patients found that on successful induction of clinical remission with EEN, intermittent EEN for 2 weeks, every 8 weeks, in absence of immunomodulators or biologics, maintained remission compared with patients on free diet with EN supplementation (25\% of calories) (HR 0.48, $\mathrm{p}=0.0051) .{ }^{67}$ Also mucosal healing was achieved by $52 \%$ of intermittent EEN patients at 12 months. This maintenance strategy, however, was not compared with immunomodulator/biological maintenance, which most centres practice. Similar studies in adults are lacking.

Partial EN (PEN) involves supplying at least half of a patient's caloric intake in the form of EN with the rest of the caloric intake from a whole food diet. The distinction between PEN and nutritious supplementation is somewhat blurred. Paediatric studies have found PEN to be effective at maintaining clinical remission. ${ }^{68}{ }^{69} \mathrm{PEN}$ has also been demonstrated to maintain remission among adult patients with CD. A meta-analysis of nine studies found that PEN was associated with higher rates of clinical remission compared with unrestricted diet $(\mathrm{p}<0.05) .^{70}$ However, all studies in this meta-analysis were conducted in Japan. Despite these positive results, further studies are needed to investigate the efficacy of PEN and intermittent EEN as maintenance therapy in adults.

\section{EEN and biological drug treatment}

Biological drugs, including anti-TNF agents, cell trafficking inhibitors and interleukin-12/23 blockers, are potent in inducing remission in CD. There is currently no RCT which compares EEN with biologics in adults. An RCT of 26 paediatric patients found comparable efficacy of EEN (83.3\%) to infliximab (90.9\%) in inducing clinical remission. ${ }^{71}$ In countries where public and private healthcare regulations permit a top-down approach, biological drugs have often become first-line therapy for CD. In certain countries, however, public and/or private healthcare regulations mandate failure of other agents before a biological drug is permitted. Despite the availability of biologics, EEN may still play a role in both these healthcare settings.

EEN administration during biological induction may reduce risk of treatment failure. Meta-analysis of four adult studies comparing the remission rates of concomitant EEN and infliximab versus infliximab alone found significantly improved long-term clinical remission rates with concomitant therapy $(74.5 \%$ vs $49.2 \%, \mathrm{p}<0.01){ }^{72}$ However, three studies were retrospective and assessed as moderate quality, with one prospective high-quality study. The mechanisms behind improved remission rates and infliximab persistence with concomitant EEN may involve additive anti-inflammatory effects or improved biological responsiveness in nutritionally replete patients.
It is unclear if EEN would have similar beneficial effects in combination with other biological drugs. Adding PEN to infliximab therapy in adult patients with $\mathrm{CD}$ has been shown in cohort studies to reduce disease recurrence and loss-of-response. ${ }^{73} 74$

EEN can induce remission in patients with disease refractory to biologics such as infliximab. In a retrospective study, four in six adult patients achieved clinical remission with 12 weeks of EEN after becoming refractory of infliximab. ${ }^{75}$ EEN was associated with a decrease in the mean CDAI scores (388.8 vs $160.0, \mathrm{p}<0.001$ ), and improvements in BMI, haemoglobin and albumin. Due to the small sample size and lack of control arm, these results are to be interpreted with caution. Further studies are needed, particularly with longer-term follow-up.

\section{Limitations of EEN use in adults}

A challenge in using EEN clinically is relatively high noncompliance rates. ${ }^{76}$ Adults are generally less compliant with EEN than children. ${ }^{38}$ Major reasons include the poor palatability of some formulations, lack of variety, missing out on the social aspects of food, lack of motivation, lack of acceptance of an NGT, financial costs and the length of treatment. ${ }^{6}$ Children do not have autonomy over their healthcare decisions. A parent or guardian can often decide for a particular treatment despite a child's protest. This may be one reason why EEN has seen more success in paediatric trials.

In general, rates of compliance to EEN therapy range from $50 \%$ to $80 \% .{ }^{17}$ Compliance rates reduce with longer durations of therapy. ${ }^{76}$ Adherence to EEN is influenced by support received by patients during the therapy timeframe. High rates of adherence of around $80 \%$ were observed in studies in which patients received in-patient EEN and had shorter EEN therapy durations. In one RCT comparing EEN to corticosteroids, 57\% discontinued EEN treatment, with majority $(69 \%)$ citing poor palatability as their reason. ${ }^{77} \mathrm{~A}$ systematic review of EN that was not restricted to $\mathrm{CD}$, similarly found highest adherence rates in hospital settings with support and monitoring from nursing staff and nutritionists. ${ }^{37}$ Older, less palatable elemental formulations resulted in lower compliance. ${ }^{3637}$

Lack of support in the outpatient setting is a major barrier to the more widespread uptake of EEN in the treatment of adult patients with CD. Several studies have attempted to address this. Piquet et al attributed their high adherence rate of close to $80 \%$ to having the staffing and infrastructure to provide nutritional support. ${ }^{78} \mathrm{Kraft}$ et al also found patient education regarding the benefits of EEN improves compliance. ${ }^{79}$ Due to poor compliance among adults, most adult CD guidelines advise EEN be reserved for when corticosteroid are ineffective or contraindicated. ${ }^{14}$ In countries such as Japan, guidelines recommend the use of EEN as first-line treatment for inducing remission in adult $\mathrm{CD} .{ }^{80}$ The difference of EEN uptake between countries could relate to cultural differences. Further formula developments to address palatability and 
offer more variety may increase compliance. Education programmes for both patients and health staff provide safer avenues for EEN use and promotes patient compliance. ${ }^{81}$ Addressing these factors may enhance uptake of EEN as routine CD therapy among adult patients in Western countries.

\section{EEN use in current practice}

A multidisciplinary approach is recommended in commencing a patient on EEN. A discussion between the patient, treating specialist and dietitian is advised to assess suitability for EEN. ${ }^{82}$ Likelihood of compliance, and risks and benefits of using EEN in place of, or in addition to, other $\mathrm{CD}$ treatments needs to be considered. The dietitian assesses for malnutrition and calculates the recommended nutritional intake for the patient. Presence of malnutrition requires gradual up titration of EEN with electrolyte monitoring to reduce risk of refeeding syndrome. Formulations can then be selected to meet the specific needs of the patient. The largest resource barrier in using EEN is the need for institutional patient support throughout the treatment to ensure compliance and safety. ${ }^{38}$ EEN is usually initiated orally with a polymeric formulation. Oral feeding has been associated with better quality of life (QOL) while preserving a similar efficacy as NGT delivery. ${ }^{84} 85$

The duration of treatment for induction of remission varies from 10 days to 12 weeks, however, most institutions practise a duration of $6-8$ weeks. A recent retrospective study found that EEN treatment durations of 6 weeks or greater were associated with improved efficacy. ${ }^{86}$ The volume of EEN formula supplied to patients also varies depending on the macronutrient and energy requirements of the individual. Gradual uptitration is recommended when adherence is an issue. ${ }^{82}$

There is no set definition for what a clinical response constitutes and would vary between countries and institutions. This may involve monitoring decreases in patientreported outcomes, HBI or CDAI scores. Guidelines by Day et al recommend alternative treatment options if clinical responses has not been achieved after 2 weeks of EEN. ${ }^{82}$ The latest Selecting Therapeutic Targets in Inflammatory Bowel Disease guidelines delineate clinical response as an immediate treatment goal in $\mathrm{CD}$, clinical remission and CRP normalisation as an intermediate-term treatment goal, and achievement of endoscopic healing, absence of disability and normalisation of health-related QOL as a long-term treatment goal. ${ }^{87}$

Patients who achieve remission are typically continued on EEN for a minimum of 6-8 weeks. Following this, whole foods are slowly reintroduced. One recommendation is to reintroduce food over a 5-day period with one additional meal introduced to their diet each day as EEN is weaned. ${ }^{88}$ Another option on weaning EEN is to transition to maintenance PEN and/or other restrictive diets. CDTEAT is a whole food diet that aims to mimics the composition of EEN. A study found CDTREAT has similar effects on the gut microbiota in adults while improving compliance compared with EEN, while in children $4 / 5$ had a response and $3 / 5$ entered clinical remission. ${ }^{89}$ The CD exclusion diet (CDED) is another popular diet that has been used alone or in combination with PEN. It aims to exclude dietary factors that have evidence for IBD onset or exacerbation from a combination of human epidemiological and animal trials. In an RCT of CD children, CDED in combination with PEN was found to have equivalent rates of clinical remission as EEN for 6 weeks followed by PEN, however, with improved compliance and sustained remission at 12 weeks. ${ }^{90}$ An uncontrolled observational study has also shown high success in mixed adult and paediatric CD populations treated with CDED and PEN.$^{30}$ Both CDTREAT and CDED can be an alternative to EEN or a longer-term maintenance strategy post-EEN with or without PEN.

\section{CONCLUSION}

Although first-line in paediatric patients, EEN has also shown promise in the management of adult patient with CD. In adults, evidence suggests that EEN has similar remission rates as corticosteroids. EEN avoids the metabolic and systemic immunosuppressive effects of corticosteroids. In addition, its combination with infliximab may improve induction and persistence of remission. Benefits of combining EEN with other biologics is less clear. In structuring and penetrating disease, EEN can avoid the need for surgery in a subset of patients, and improve outcomes in those who eventually undergo surgery. EEN trial evidence is often limited by small patient numbers, lack of blinding, high non-compliance rates and difficulties with meta-analysis due to variability in study protocols. Despite this, EEN should be considered in certain patients in a modern-day CD treatment. Larger, higherquality studies are needed to confirm these benefits in adults. Infrastructure to support patients may improve adherence in future trials.

Twitter Nikola Mitrev nikola.m@hotmail.com

Contributors VCK: study supervision. HH, NM and VCK: literature search and review. $\mathrm{HH}, \mathrm{NM}$ and VCK: manuscript drafting. $\mathrm{HH}, \mathrm{NM}, \mathrm{BH}$ and VCK: critical revision.

Funding The authors have not declared a specific grant for this research from any funding agency in the public, commercial or not-for-profit sectors.

Competing interests None declared.

Patient consent for publication Not applicable.

Provenance and peer review Not commissioned; externally peer reviewed.

Data availability statement Data are available in a public, open access repository.

Supplemental material This content has been supplied by the author(s). It has not been vetted by BMJ Publishing Group Limited (BMJ) and may not have been peer-reviewed. Any opinions or recommendations discussed are solely those of the author(s) and are not endorsed by BMJ. BMJ disclaims all liability and responsibility arising from any reliance placed on the content. Where the content includes any translated material, BMJ does not warrant the accuracy and reliability of the translations (including but not limited to local regulations, clinical guidelines, terminology, drug names and drug dosages), and is not responsible for any error and/or omissions arising from translation and adaptation or otherwise.

Open access This is an open access article distributed in accordance with the Creative Commons Attribution Non Commercial (CC BY-NC 4.0) license, which 
permits others to distribute, remix, adapt, build upon this work non-commercially, and license their derivative works on different terms, provided the original work is properly cited, appropriate credit is given, any changes made indicated, and the use is non-commercial. See: http://creativecommons.org/licenses/by-nc/4.0/.

ORCID iD

Nikola Mitrev http://orcid.org/0000-0001-9273-7445

\section{REFERENCES}

1 Loftus EV. Clinical epidemiology of inflammatory bowel disease: incidence, prevalence, and environmental influences. Gastroenterology 2004;126:1504-17.

2 Feuerstein JD, Cheifetz AS. Crohn disease: epidemiology, diagnosis, and management. Mayo Clin Proc 2017;92:1088-103.

3 Liu J, Kariyawasam V, Borody T. IDDF2018-ABS-0034 High agespecific prevalence of inflammatory bowel disease amongst the elderly in the city of canada bay area, sydney: a metropolitan, population-based study. Gut 2018;67:A35.

4 Cheifetz AS. Management of active Crohn disease. JAMA 2013;309:2150-8.

5 van Rheenen PF, Aloi M, Assa A, et al. The medical management of paediatric Crohn's disease: an ECCO-ESPGHAN guideline update. J Crohns Colitis 2020. doi:10.1093/ecco-jcc/jjaa161. [Epub ahead of print: 07 Oct 2020].

6 Wall CL, Day AS, Gearry RB. Use of exclusive enteral nutrition in adults with Crohn's disease: a review. World J Gastroenterol 2013:19:7652-60.

$7 \mathrm{Yu}$ Y, Chen K-C, Chen J. Exclusive enteral nutrition versus corticosteroids for treatment of pediatric Crohn's disease: a metaanalysis. World J Pediatr 2019;15:26-36.

8 Heuschkel RB, Menache CC, Megerian JT, et al. Enteral nutrition and corticosteroids in the treatment of acute Crohn's disease in children. J Pediatr Gastroenterol Nutr 2000;31:8-15.

9 Soo J, Malik BA, Turner JM, et al. Use of exclusive enteral nutrition is just as effective as corticosteroids in newly diagnosed pediatric Crohn's disease. Dig Dis Sci 2013;58:3584-91.

10 Thomas AG, Taylor F, Miller V. Dietary intake and nutritional treatment in childhood Crohn's disease. J Pediatr Gastroenterol Nutr 1993;17:75-81.

11 Borrelli O, Cordischi L, Cirulli M, et al. Polymeric diet alone versus corticosteroids in the treatment of active pediatric Crohn's disease: a randomized controlled open-label trial. Clin Gastroenterol Hepatol 2006:4:744-53.

12 Bischoff SC, Escher J, Hébuterne X, et al. ESPEN practical guideline: clinical nutrition in inflammatory bowel disease. Clin Nutr 2020;39:632-53.

13 Prantera C, Marconi S. Glucocorticosteroids in the treatment of inflammatory bowel disease and approaches to minimizing systemic activity. Therap Adv Gastroenterol 2013;6:137-56.

14 Alex G. Clinical update for general practitioners and physicians inflammatory bowel disease. Melbourne: GESA, 2018.

15 Lamb CA, Kennedy NA, Raine T, et al. British Society of gastroenterology consensus guidelines on the management of inflammatory bowel disease in adults. Gut 2019;68:s1-s106.

16 Costa-Santos MP, Palmela C, Torres J, et al. Preoperative enteral nutrition in adults with complicated Crohn's disease: effect on disease outcomes and gut microbiota. Nutrition 2020;70S:100009.

17 Goh J, O'Morain CA. Review article: nutrition and adult inflammatory bowel disease. Aliment Pharmacol Ther 2003;17:307-20.

18 Donnellan CF, Yann LH, Lal S. Nutritional management of Crohn's disease. Therap Adv Gastroenterol 2013;6:231-42.

19 Klaus J, Armbrecht G, Steinkamp M, et al. High prevalence of osteoporotic vertebral fractures in patients with Crohn's disease. Gut 2002;51:654-8.

20 Nguyen GC, Munsell M, Harris ML. Nationwide prevalence and prognostic significance of clinically diagnosable protein-calorie malnutrition in hospitalized inflammatory bowel disease patients. Inflamm Bowel Dis 2008:14:1105-11.

21 Voitk AJ, Echave V, Feller JH, et al. Experience with elemental diet in the treatment of inflammatory bowel disease. is this primary therapy? Arch Surg 1973;107:329-33.

22 Keetarut K, Whitley L, Murray C. P-114: Response to exclusive enteral nutrition (EEN) in adolescent patients with newly diagnosed Crohn's disease (CD). J Crohns Colitis 2014.

23 Grover Z, Lewindon P. Two-Year outcomes after exclusive enteral nutrition induction are superior to corticosteroids in pediatric
Crohn's disease treated early with thiopurines. Dig Dis Sci 2015;60:3069-74.

24 Duboc H, Rajca S, Rainteau D, et al. Connecting dysbiosis, bileacid dysmetabolism and gut inflammation in inflammatory bowel diseases. Gut 2013;62:531-9.

25 Alhagamhmad MH, Day AS, Lemberg DA, et al. An overview of the bacterial contribution to Crohn disease pathogenesis. J Med Microbiol 2016;65:1049-59.

26 Leach ST, Mitchell HM, Eng WR, et al. Sustained modulation of intestinal bacteria by exclusive enteral nutrition used to treat children with Crohn's disease. Aliment Pharmacol Ther 2008;28:724-33.

27 Guinet-Charpentier C, Lepage P, Morali A, et al. Effects of enteral polymeric diet on gut microbiota in children with Crohn's disease. Gut 2017;66:194-5.

28 Lionetti P, Callegari ML, Ferrari S, et al. Enteral nutrition and microflora in pediatric Crohn's disease. JPEN J Parenter Enteral Nutr 2005;29:S173-5; discussion S175-8, S184-8.

29 Diederen K, Li JV, Donachie GE, et al. Exclusive enteral nutrition mediates gut microbial and metabolic changes that are associated with remission in children with Crohn's disease. Sci Rep 2020;10:18879.

30 Sigall Boneh R, Sarbagili Shabat C, Yanai H, et al. Dietary therapy with the Crohn's disease exclusion diet is a successful strategy for induction of remission in children and adults failing biological therapy. J Crohns Colitis 2017;11:1205-12.

31 Nahidi L, Day AS, Lemberg DA, et al. Differential effects of nutritional and non-nutritional therapies on intestinal barrier function in an in vitro model. $J$ Gastroenterol 2012;47:107-17.

32 Ong C, Lim PT, Logarajah V, et al. Exclusive enteral nutrition with concomitant early thiopurine use was effective in maintaining steroid-free remission in a Southeast Asian cohort of children with Crohn's disease. BMC Gastroenterol 2018;18:185.

33 de Bie C, Kindermann A, Escher J. Use of exclusive enteral nutrition in paediatric Crohn's disease in the Netherlands. J Crohns Colitis 2013;7:263-70.

34 Wall CL, Gearry RB, Day AS. Treatment of Active Crohn's Disease with Exclusive and Partial Enteral Nutrition: A Pilot Study in Adults. Inflamm Intest Dis 2018;2:219-27.

35 Rigaud D, Cosnes J, Le Quintrec Y, et al. Controlled tria comparing two types of enteral nutrition in treatment of active Crohn's disease: elemental versus polymeric diet. Gut 1991;32:1492-7.

36 Rodrigues AF, Johnson T, Davies P, et al. Does polymeric formula improve adherence to liquid diet therapy in children with active Crohn's disease? Arch Dis Child 2007;92:767-70.

37 Gea Cabrera A, Sanz-Lorente M, Sanz-Valero J, et al. Compliance and adherence to enteral nutrition treatment in adults: a systematic review. Nutrients 2019;11. doi:10.3390/nu11112627. [Epub ahead of print: 02 Nov 2019].

38 Narula N, Dhillon A, Zhang D, et al. Enteral nutritional therapy for induction of remission in Crohn's disease. Cochrane Database Syst Rev 2018;4:CD000542.

39 Logan M, Gkikas K, Svolos V, et al. Analysis of 61 exclusive enteral nutrition formulas used in the management of active Crohn's disease-new insights into dietary disease triggers. Aliment Pharmacol Ther 2020;51:935-47.

40 Limketkai BN, Iheozor-Ejiofor Z, Gjuladin-Hellon T, et al. Dietary interventions for induction and maintenance of remission in inflammatory bowel disease. Cochrane Database Syst Rev 2019;2:CD012839.

41 D'Haens G. Mucosal healing in pediatric Crohn's disease: the goal of medical treatment. Inflamm Bowel Dis 2004:10:479-80.

42 Grover Z, Burgess C, Muir R, et al. Early mucosal healing with exclusive enteral nutrition is associated with improved outcomes in newly diagnosed children with luminal Crohn's disease. J Crohns Colitis 2016;10:1159-64.

43 Modigliani R, Mary J-Y, Simon J-F. Clinical, biological, and endoscopic picture of attacks of Crohn's disease: Evolution on prednisolone. Gastroenterology 1990;98:811-8.

44 Chen J-M, He L-W, Yan T, et al. Oral exclusive enteral nutrition induces mucosal and transmural healing in patients with Crohn's disease. Gastroenterol Rep 2019;7:176-84.

45 Schwab D, Raithel M, Hahn EG. [Enteral nutrition in acute Crohn disease]. Z Gastroenterol 1998;36:983-95.

46 Sharma S, Gupta A, Kedia S, et al. Efficacy and tolerability of exclusive enteral nutrition in adult patients with complicated Crohn's disease. Intest Res 2021;19:291-300.

47 Yang Q, Li M, Yao J. Exclusive enteral nutrition is effective for female Crohn's disease patients who are pregnant or preparing for pregnancy. Digestive Diseases Week, 2020. 
48 Frivolt K, Schwerd T, Werkstetter KJ, et al. Repeated exclusive enteral nutrition in the treatment of paediatric Crohn's disease: predictors of efficacy and outcome. Aliment Pharmacol Ther 2014;39:1398-407.

49 Yan D, Ren J, Wang G, et al. Predictors of response to enteral nutrition in abdominal enterocutaneous fistula patients with Crohn's disease. Eur J Clin Nutr 2014;68:959-63.

50 Matsueda K. Therapeutic efficacy of elemental enteral alimentation in Crohn's disease. J Gastroenterol 2000;35 Suppl 12:19.

$51 \mathrm{Hu}$ D, Ren J, Wang G, et al. Exclusive enteral nutritional therapy can relieve inflammatory bowel stricture in Crohn's disease. J Clin Gastroenterol 2014;48:790-5.

52 Yang Q, Gao X, Chen H, et al. Efficacy of exclusive enteral nutrition in complicated Crohn's disease. Scand J Gastroenterol 2017;52:995-1001.

53 Rieder F, Latella G, Magro F, et al. European Crohn's and colitis organisation topical review on prediction, diagnosis and management of Fibrostenosing Crohn's disease. J Crohns Colitis 2016;10:873-85.

54 Subramanian V, Saxena S, Kang J-Y, et al. Preoperative steroid use and risk of postoperative complications in patients with inflammatory bowel disease undergoing abdominal surgery. Am J Gastroenterol 2008;103:2373-81.

55 Brennan GT, Ha I, Hogan C, et al. Does preoperative enteral or parenteral nutrition reduce postoperative complications in Crohn's disease patients: a meta-analysis. Eur J Gastroenterol Hepatol 2018;30:997-1002

56 Li G, Ren J, Wang G, et al. Preoperative exclusive enteral nutrition reduces the postoperative septic complications of fistulizing Crohn's disease. Eur J Clin Nutr 2014:68:441-6.

57 Li Y, Zuo L, Zhu W, et al. Role of exclusive enteral nutrition in the preoperative optimization of patients with Crohn's disease following immunosuppressive therapy. Medicine 2015;94:e478.

58 Ge X, Tang S, Yang X, et al. The role of exclusive enteral nutrition in the preoperative optimization of laparoscopic surgery for patients with Crohn's disease: a cohort study. Int J Surg 2019;65:39-44.

59 Heerasing $\mathrm{N}$, Thompson B, Hendy $\mathrm{P}$, et al. Exclusive enteral nutrition provides an effective bridge to safer interval elective surgery for adults with Crohn's disease. Aliment Pharmacol Ther 2017:45:660-9.

60 Zheng X-B, Peng X, Xie X-Y, et al. Enteral nutrition is associated with a decreased risk of surgical intervention in Crohn's disease patients with spontaneous intra-abdominal abscess. Rev Esp Enferm Dig 2017;109:834-42.

61 Mitrev N, Lai R, Nguyen TMP. Exclusive enteral nutrition associated with reduced short-term surgery rates in adult Crohn's disease: local experiences of two IBD services. Australian Gastroenterology Week, 2019.

62 Beaupel N, Brouquet A, Abdalla S, et al. Preoperative oral polymeric diet enriched with transforming growth factor-beta 2 (Modulen) could decrease postoperative morbidity after surgery for complicated ileocolonic Crohn's disease. Scand J Gastroenterol 2017;52:5-10.

63 Wang H, Zuo L, Zhao J, et al. Impact of preoperative exclusive enteral nutrition on postoperative complications and recurrence after bowel resection in patients with active Crohn's disease. World J Surg 2016;40:1993-2000.

64 Yamamoto T, Nakahigashi M, Umegae S, et al. Impact of longterm enteral nutrition on clinical and endoscopic recurrence after resection for Crohn's disease: a prospective, non-randomized, parallel, controlled study. Aliment Pharmacol Ther 2007;25:67-72.

65 Yamamoto T, Shiraki M, Nakahigashi M, et al. Enteral nutrition to suppress postoperative Crohn's disease recurrence: a five-year prospective cohort study. Int J Colorectal Dis 2013;28:335-40.

66 Esaki M, Matsumoto T, Hizawa K, et al. Preventive effect of nutritional therapy against postoperative recurrence of Crohn disease, with reference to findings determined by intra-operative enteroscopy. Scand J Gastroenterol 2005;40:1431-7.

67 Pigneur Arnaud B, Martinez-Vinson C, Bourmaud A, et al. OP15 Cyclic exclusive enteral nutrition to maintain longterm drug-free remission in Paediatric Crohn's Disease: The CD HOPE study of the GETAID pédiatrique. J Crohns Colitis 2021;15:S015

68 Takagi S, Utsunomiya K, Kuriyama S, et al. Effectiveness of an 'half elemental diet' as maintenance therapy for Crohn's disease: A randomized-controlled trial. Aliment Pharmacol Ther 2006;24:1333-40.

69 Wilschanski M, Sherman P, Pencharz P, et al. Supplementary enteral nutrition maintains remission in paediatric Crohn's disease. Gut 1996;38:543-8.

70 Hirai F, Takeda T, Takada Y, et al. Efficacy of enteral nutrition in patients with Crohn's disease on maintenance anti-TNF- alpha antibody therapy: a meta-analysis. J Gastroenterol 2020;55:133-41.

71 Luo Y, Yu J, Lou J, et al. Exclusive enteral nutrition versus infliximab in inducing therapy of pediatric Crohn's disease. Gastroenterol Res Pract 2017;2017:6595048.

72 Nguyen DL, Palmer LB, Nguyen ET, et al. Specialized enteral nutrition therapy in Crohn's disease patients on maintenance infliximab therapy: a meta-analysis. Therap Adv Gastroenterol 2015;8:168-75.

73 Sazuka S, Katsuno T, Nakagawa T, et al. Concomitant use of enteral nutrition therapy is associated with sustained response to infliximab in patients with Crohn's disease. Eur J Clin Nutr 2012;66:1219-23.

74 Hirai $\mathrm{F}$, Ishihara $\mathrm{H}$, Yada S, et al. Effectiveness of concomitant enteral nutrition therapy and infliximab for maintenance treatment of Crohn's disease in adults. Dig Dis Sci 2013;58:1329-34.

75 Sood A, Singh A, Sudhakar R, et al. Exclusive enteral nutrition for induction of remission in anti-tumor necrosis factor refractory adult Crohn's disease: the Indian experience. Intest Res 2020;18:184-91.

76 Stow R, Ives N, Smith C, et al. A cluster randomised feasibility trial evaluating nutritional interventions in the treatment of malnutrition in care home adult residents. Trials 2015;16:433.

77 Malchow H, Steinhardt HJ, Lorenz-Meyer H, et al. Feasibility and effectiveness of a defined-formula diet regimen in treating active Crohn's disease. European cooperative Crohn's disease study III. Scand J Gastroenterol 1990;25:235-44.

78 Piquet M-A, Ozsahin M, Larpin I, et al. Early nutritional intervention in oropharyngeal cancer patients undergoing radiotherapy. Support Care Cancer 2002;10:502-4.

79 Kraft M, van den Berg N, Kraft K, et al. Development of a telemedical monitoring concept for the care of malnourished geriatric home-dwelling patients: a pilot study. Maturitas 2012;72:126-31.

80 Matsuoka K, Kobayashi T, Ueno F, et al. Evidence-Based clinical practice guidelines for inflammatory bowel disease. J Gastroenterol 2018;53:305-53.

81 Boullata JI, Carrera AL, Harvey L, et al. ASPEN Safe Practices for Enteral Nutrition Therapy [Formula: see text]. JPEN J Parenter Enteral Nutr 2017;41:15-103.

82 Day A, Wood J, Melton S, et al. Exclusive enteral nutrition: an optimal care pathway for use in adult patients with active Crohn's disease. JGH Open 2020;4:260-6.

83 Allison SP. Some psychological and physiological aspects of enteral nutrition. Gut 1986;27 Suppl 1:18-24.

84 Rubio A, Pigneur B, Garnier-Lengliné H, et al. The efficacy of exclusive nutritional therapy in paediatric Crohn's disease, comparing fractionated oral vs. continuous enteral feeding. Aliment Pharmacol Ther 2011;33:1332-9.

85 Gailhoustet L, Goulet O, Cachin N, et al. [Study of psychological repercussions of 2 modes of treatment of adolescents with Crohn's disease]. Arch Pediatr 2002;9:110-6.

86 Ramaswamy PK, Mutsekwa R, Lan X. Exclusive Enteral Nutrition with a polymeric diet is efficacious in inducing remission in adults with active Crohn's disease. Digestive Diseases Week, 2020.

87 Turner D, Ricciuto A, Lewis A, et al. STRIDE-II: an update on the selecting therapeutic targets in inflammatory bowel disease (STRIDE) initiative of the International organization for the study of IBD (IOIBD): determining therapeutic goals for Treat-to-Target strategies in IBD. Gastroenterology 2021;160:1570-83.

88 Faiman A, Mutalib M, Moylan A, et al. Standard versus rapid food reintroduction after exclusive enteral nutritional therapy in paediatric Crohn's disease. Eur J Gastroenterol Hepatol 2014;26:276-81.

89 Svolos V, Hansen R, Nichols B, et al. Treatment of active Crohn's disease with an ordinary food-based diet that replicates exclusive enteral nutrition. Gastroenterology 2019;156:1354-67.

90 Levine A, Wine E, Assa A, et al. Crohn's disease exclusion diet plus partial enteral nutrition induces sustained remission in a randomized controlled trial. Gastroenterology 2019;157:440-50.

91 Engelman JL BL, Murphy GM, Sladen GE. Comparison of a semi elemental diet (Peptamen) with prednisolone in the primarytreatment of active ileal Crohn's disease. Gastroenterology 1993;104:A697.

92 Gassull MA, Fernández-Bañares F, Cabré E, et al. Fat composition may be a clue to explain the primary therapeutic effect of enteral nutrition in Crohn's disease: results of a double blind randomised multicentre European trial. Gut 2002;51:164-8.

93 González-Huix F, de León R, Fernández-Bañares F, et al. Polymeric enteral diets as primary treatment of active Crohn's disease: a prospective steroid controlled trial. Gut 1993;34:778-82. 
94 Gorard DA, Hunt JB, Payne-James JJ, et al. Initial response and subsequent course of Crohn's disease treated with elemental diet or prednisolone. Gut 1993;34:1198-202.

95 Lindor KD, Fleming CR, Burnes JU, et al. A randomized prospective trial comparing a defined formula diet, corticosteroids, and a defined formula diet plus corticosteroids in active Crohn's disease. Mayo Clin Proc 1992;67:328-33.

96 Lochs H, Steinhardt HJ, Klaus-Wentz B, et al. Comparison of enteral nutrition and drug treatment in active Crohn's disease. Results of the European cooperative Crohn's disease study. IV. Gastroenterology 1991;101:881-8.
97 Mantzaris GJ AE, Amperiadis P, Kourtessas D. A randomized prospective trial in active Crohn's disease comparing a polymeric diet, prednisolone, and a polymeric diet plus prednisolone. Gastroenterology 1996;110.

98 Okada M, Yao T, Yamamoto T, et al. Controlled trial comparing an elemental diet with prednisolone in the treatment of active Crohn's disease. Hepatogastroenterology 1990;37:72-80.

99 O'Morain C, Segal AW, Levi AJ. Elemental diet as primary treatment of acute Crohn's disease: a controlled trial. BMJ 1984;288:1859-62.

100 Zoli G, Carè M, Parazza M, et al. A randomized controlled study comparing elemental diet and steroid treatment in Crohn's disease. Aliment Pharmacol Ther 1997;11:735-40. 